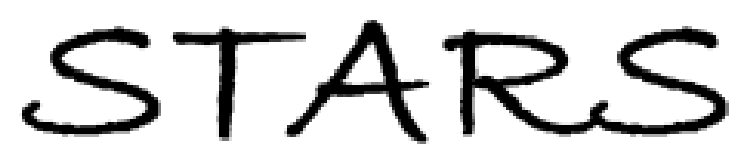

University of Central Florida

STARS

Faculty Bibliography 2010s

Faculty Bibliography

$1-1-2011$

\title{
Mapping the mechanical action of light
}

Dana C. Kohlgraf-Owens

Sergey Sukhov

University of Central Florida

Aristide Dogariu

University of Central Florida

Find similar works at: https://stars.library.ucf.edu/facultybib2010

University of Central Florida Libraries http://library.ucf.edu

This Article is brought to you for free and open access by the Faculty Bibliography at STARS. It has been accepted for inclusion in Faculty Bibliography 2010s by an authorized administrator of STARS. For more information, please contactSTARS@ucf.edu.

\section{Recommended Citation}

Kohlgraf-Owens, Dana C.; Sukhov, Sergey; and Dogariu, Aristide, "Mapping the mechanical action of light" (2011). Faculty Bibliography 2010s. 1490.

https://stars.library.ucf.edu/facultybib2010/1490

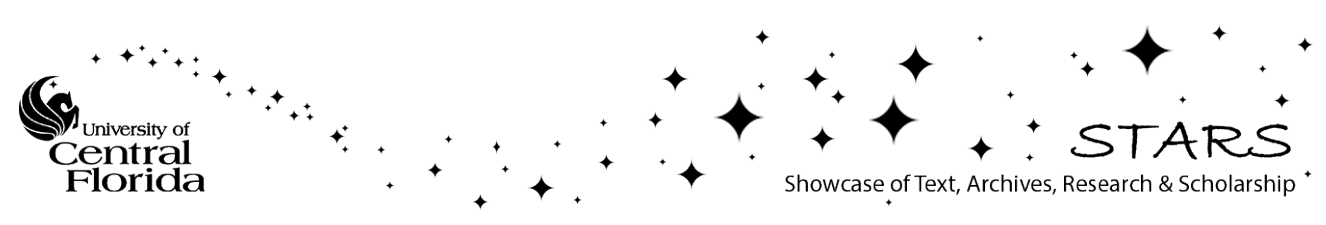




\title{
Mapping the mechanical action of light
}

\author{
Dana C. Kohlgraf-Owens, Sergey Sukhov, and Aristide Dogariu \\ CREOL, The College of Optics and Photonics, University of Central Florida, \\ Orlando, Florida 32816, USA
}

(Received 7 March 2011; revised manuscript received 25 May 2011; published 29 July 2011)

\begin{abstract}
We demonstrate that the mechanical action of light manifests itself in the perceived topography measured with a scanning probe microscope. This modality of sensing optically induced forces opens up possibilities to quantify properties of electromagnetic fields.
\end{abstract}

DOI: 10.1103/PhysRevA.84.011807

PACS number(s): 42.50.Wk, 41.20.-q, 07.79.-v

Scanning probe microscopies are used to probe a variety of surface and subsurface properties because of their high sensitivity and high spatial resolution. For instance, atomic force microscopy (AFM) is used to measure forces on the order of piconewtons or less with nanometric resolution. Typically, a small probe is scanned either vertically to generate force-distance curves or laterally to map a desired physical quantity. In a similar manner, the intensity of a light field can be measured with a near-field scanning optical microscope (NSOM) either by collecting light with a subwavelength aperture or by collecting the scattering off of a subwavelength probe (s-NSOM). In contrast, it is well known that light can also induce mechanical action on material objects [1,2] and therefore a scanning probe may be used to detect such forces. When performing a standard scan, the optical force will manifest itself as a topographic signal. In this paper we will show that this optically induced topography can be used to quantitatively determine the strength of the optical force interaction with the probe.

The use of scanning probe microscopes to measure external forces such as van der Waals, meniscus, electrostatic, and magnetic has been extensively documented in the literature $[3,4]$. However, the possibility to measure optically induced forces has been explored only theoretically [5] and in some qualitative experiments [6-9]. Similarly, the tuning fork itself, which is the usual component of scanning probe microscopes, has been suggested as a sensor of electromagnetic radiation [9].

Here we demonstrate that using a scanning probe microscope, the optically induced forces on that probe can be quantitatively determined. As we will show, significant optical forces are observed for moderate input light intensities. Consequently, the influence of optical forces should be carefully considered in more traditional NSOM measurements, where the presence of a sharp metal-coated tip can generate strong-localfield gradients, which may modify the perceived topography.

We will demonstrate this concept by presenting two different experiments. First, we will illustrate the detection of the optically induced forces due to propagating radiation using a dielectric probe. Second, we will show that one can also detect the mechanical action of evanescent fields using a standard NSOM tip even for moderate illumination intensities. Let us note that the situation described in the present paper should be distinguished from the case of so-called photonic force microscopy, where an optically trapped particle is used as a force sensor [10]. This technique relies on the presence of Brownian motion to build up statistics of the particle locations and is fundamentally different from the deterministic procedure we present here.

To measure the influence of the optical forces on a probe, we perform scans over the core of a single-mode fiber with its exit surface cleaved and polished normal to the optical axis. Laser radiation of different powers is coupled into the fiber with an effective mode diameter of $3.5 \mu \mathrm{m}$. The probe is an uncoated tapered glass capillary tube with a 100-nm-diam aperture mounted on a Nanonics MV4000 scanner. Because optically induced forces affect the scanner's feedback mechanism, topography scans are sensitive to optical radiation impinging on the tip. By separately measuring the "real" surface topography, we extract the optically induced contribution to the measured topography, as illustrated in Fig. 1.

Several features of these optical topographies are worth noting. First, the effect of optically induced forces manifests itself as an increase of the tip sample separation. This is because the light impinging on the probe generates a negative shift in the tuning fork resonance frequency whose strength depends on the three-dimensional field distribution and its interaction with the probe. The feedback compensates for the additional optically induced frequency shift by retracting the tip to reduce the contribution of surface forces, thereby maintaining a constant resonance frequency shift on the tuning fork. One can notice that the effect of the light on the probe is strongest in the upper left corner of the scan, which corresponds to tip positions past the core of the fiber. This occurs because the tip is tilted with respect to the surface as seen in the inset of Fig. 1(a); thus a larger volume of the probe is exposed to the optical field as it passes over the core resulting in a larger force.

To explain the effect illustrated in Fig. 1, we model the behavior of our tip as a damped driven harmonic oscillator [3]. In the proximity of an interface, the probe is in general affected by different types of interaction forces [3] that cause the resonance frequency of the tuning fork to shift and introduces an additional damping mechanism. These two effects result in changes of both the amplitude and the phase of the tip oscillation relative to the driving signal; the system's feedback acts to maintain this phase constant.

Because external contributions to the damping are insignificant in our experiments, we can analyze the probe oscillation based on the induced resonance frequency shift $\Delta \omega$ in a harmonic oscillator of mass $m$ that has a resonance frequency $\omega_{0}^{2}=k / m$ determined by its spring constant $k$. The dependence between the frequency shift $\Delta \omega$ and the force 

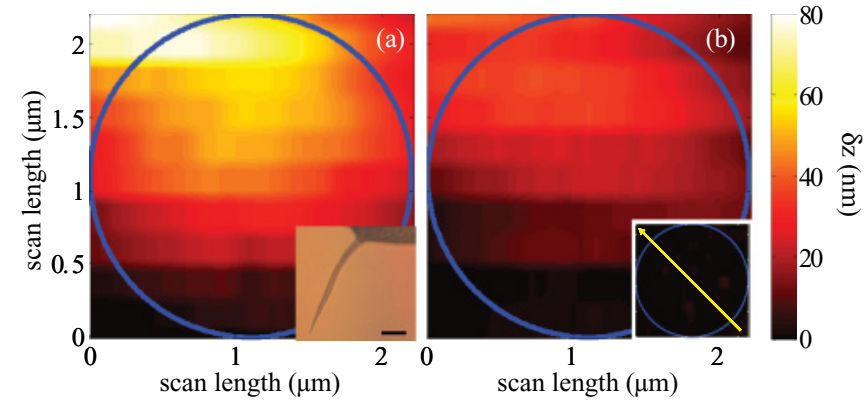

FIG. 1. (Color online) Perceived topography over the core of a single-mode optical fiber when (a) 24 and (b) $16 \mathrm{~mW}$ of 532-nm laser light is coupled into the fiber. The inset in (a) is the side view of the probe; the scale bar corresponds to $100 \mu \mathrm{m}$. The inset in (b) shows the measured topography of the fiber face. The circles indicate the location of the fiber core, which is approximately $2.2 \mu \mathrm{m}$ in diameter. The arrow indicates the orientation of the tip during scanning.

acting on the tip $F_{\text {tip }}(z)$ can be calculated as a first-order perturbation using the Hamilton-Jacobi approach [11] $\Delta \omega=$ $-\left(\omega_{0} / k A^{2}\right)\left\langle F_{\text {tip }}(z) x(t)\right\rangle$, where $x(t)$ is the unperturbed motion of tip in the absence of $F_{\text {tip }}(z)$ and averaging is performed over an entire oscillation cycle.

In this experiment, the dominant interaction between the surface and the probe is through dispersion forces [12]. When the tip is also under the influence of an external electromagnetic field, additional forces due to radiation pressure, gradient of intensity or phase, optical binding, photophoretic forces (local heating), and photoinduced stress may be present $[6,8]$. To isolate the optical effect, we used a purely dielectric system such that significant thermal, electrostatic, and magnetic effects can be avoided, leaving two main external contributions to the total force acting on the probe: the retarded van der Waals force $F_{W}$ and the mechanical action of light $F_{\text {opt }}$.

When the feedback is engaged, a constant resonance frequency shift

$$
\Delta \omega_{\mathrm{opt}}=\Delta \omega_{W}-\Delta \omega_{W-\mathrm{opt}}
$$

is maintained. In Eq. (1), $\Delta \omega_{W}$ and $\Delta \omega_{W-\text { opt }}$ represent the frequency shifts the feedback operates on when the incident radiation is turned off and on, respectively. To derive analytic equations for these terms, we model the tip-sample geometry as a silica sphere in air interacting with a silica plane. This approach is common for estimation of van der Waals forces in AFM and is valid because the radius of tip is quite large $(\sim 150 \mathrm{~nm})$ and therefore the influence on the bulk of the tip is negligible at typical tip-sample separations. We emphasize that this model is only used for the estimate of the van der Waals forces. Accounting for the large variation of the interaction forces over the course of one tip oscillation cycle, the resulting shift in the resonance frequency is obtained as [11]

$$
\Delta \omega_{W}=\frac{\omega_{0}}{k A} \frac{2 \pi R B}{3 z^{3}}\left[F_{1}^{3,0.5}\left(\frac{-2 A}{z}\right)-F_{2}^{3,1.5}\left(\frac{-2 A}{z}\right)\right],
$$

where $F_{a}^{b, c}(z)$ is the hypergeometric function, $B$ is the retarded Hamaker constant [13], $R$ is the radius of the sphere, and $z$ is the closest tip-sample separation. The expression for $\Delta \omega_{W-\text { opt }}$ is the same as Eq. (2) except that $z$ is now replaced with

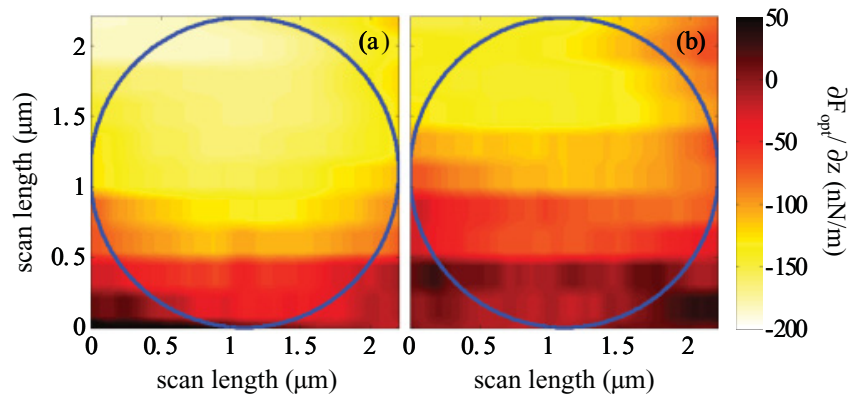

FIG. 2. (Color online) Gradient of the optical force extracted from the perceived topography shown in Fig. 1 when (a) 24- and (b) $16-\mathrm{mW}$ power is coupled into a single-mode fiber.

$z+\delta z$, where $\delta z$ describes the optically induced topography shown in Fig. 1. The retarded van der Waals force is estimated to be of the order of $10^{-8} \mathrm{~N}$ near the surface

As confirmed by numerical calculations, the distribution of the electromagnetic field emerging from the fiber changes only slightly over a distance of a few microns; consequently the longitudinal gradient of optical force is nearly constant over the range of tip oscillations considered. Thus, $\Delta \omega_{\text {opt }}$ is found to be

$$
\Delta \omega_{\mathrm{opt}}=-\frac{\omega_{0}}{2 k} \frac{\partial F_{\mathrm{opt}}}{\partial z}=\frac{-\omega_{0}}{2 k} \beta I,
$$

where $\beta$ is a constant that measures the force exerted on the tip by a unit of optical power coupled out of the fiber and $I$ is the local-field intensity at the point $(x, y)$.

Using Eqs. (1)-(3), we can obtain a direct relationship between the optically induced forces and the resulting changes in the dispersion force acting on the tip. Alternatively, we can determine the strength of the optically induced force gradients if the strength of the van der Waals contributions is known without and during the light irradiation, i.e., for separation distances $z$ and $z+\delta z$ :

$$
\frac{\partial F_{\mathrm{opt}}}{\partial z}=\frac{-4 \pi R B}{3 A}\left(\frac{1}{z^{3}} F_{W}^{3}-\frac{1}{(z+\delta z)^{3}} F_{W-\mathrm{opt}}^{3}\right),
$$

where $F_{W}^{n}=F_{1}^{n, 0.5}(-2 A / z)-F_{2}^{n, 1.5}(-2 A / z)$ and $F_{W-\mathrm{opt}}^{n}=$ $F_{1}^{n, 0.5}(-2 A /(z+\delta z))-F_{2}^{n, 1.5}(-2 A /(z+\delta z))$. Because the gradient of the dispersion force is a nonlinear function of the tip-sample separation, we require knowledge of the initial tip-sample separation at closest approach in order to determine the gradient of the optical force. Obtaining this quantity, which is typically not known, usually requires external measurements or a priori assumptions [14-16]. However because the gradient of the optical force is linear with respect to the field intensity $I(x, y)$ at each point during the scan, Eq. (4) may be used to compute the initial tip-sample separation $z$ at each location in the scan by evaluating the ratio corresponding to different input intensities.

Since our scanning probe microscope operates in intermittent contact mode, one expects a minimum tip-sample separation close to zero. Using the procedure outlined above, we obtain $z=74 \pm 1 \mathrm{~nm}$, which represents an effective tip-sample separation due to the slanted geometry of our probe. Using this value in Eq. (4), we generate maps of the measured gradients of the optically induced force $\partial F_{\text {opt }} / \partial z$. As can be seen in Fig. 2, this gradient is negative and is of the order of 
$10^{-7} \mathrm{~N} / \mathrm{m}$, comparable to the retarded van der Waals force when the tip is tens of nanometers away from the surface.

To further assess these results, we estimate the optical forces by performing numerical simulations using the finite-element method (COMSOL MULTIPHYSICS 3.5a), which account for the complex three-dimensional behavior of the optical forces near the surface and its interaction with a realistic tip. To provide an accurate representation of the tip used in the experiments, the probe was modeled based on the actual probe geometry (inset Fig. 1(a)). The length of the simulated probe was about $4 \mu \mathrm{m}$, which we expect to effectively model an infinite tip for scan points in the vicinity of the fiber core. An example of the field distribution in the cross section of the probe is shown in Fig. 3(a). Using the calculated field distribution and the Maxwell stress tensor representation [17], we then evaluated the optical force acting on the scanning probe. Note that with this approach, all the components of optical force, gradient force, radiation pressure, and force due to phase gradient [18] are taken into account.

When the probe is located $1 \mu \mathrm{m}$ past the fiber core as in Fig. 3(a), i.e., approximately where the arrow is pointing to in the inset of Fig. 1(b), we find that $F_{\text {opt }}$ is on the order of $10^{-11} \mathrm{~N}$ for a $24-\mathrm{mW}$ illuminating beam, which is comparable with typical surface forces. Calculations also show that the gradient of the optical force is nearly constant over the range of the tip oscillation considered and has a value of $-1.6 \times 10^{-7}$ $\mathrm{N} / \mathrm{m}$. Even though the field distribution varies laterally over the scan area, at each point in the scan the gradient may be assumed constant in the longitudinal direction over the range of the tip oscillation amplitude.

The electromagnetic interaction between the probe and fiber itself may, in principle, affect the field distribution and, consequently, the interaction forces. However, our systematic evaluations indicate that the effect of this so-called optical binding force $[5,6]$ that establishes between dielectric bodies irradiated by a common electromagnetic field is about two orders of magnitude weaker than the other optical forces near the surface. Thus, we expect the optical binding force to contribute insignificantly to the overall frequency shift when averaged over the large oscillation amplitude $(A \approx 90 \mathrm{~nm})$ used in our experiment.

In Fig. 3(b) we quantitatively compare the values of the optical force gradient obtained experimentally with the results
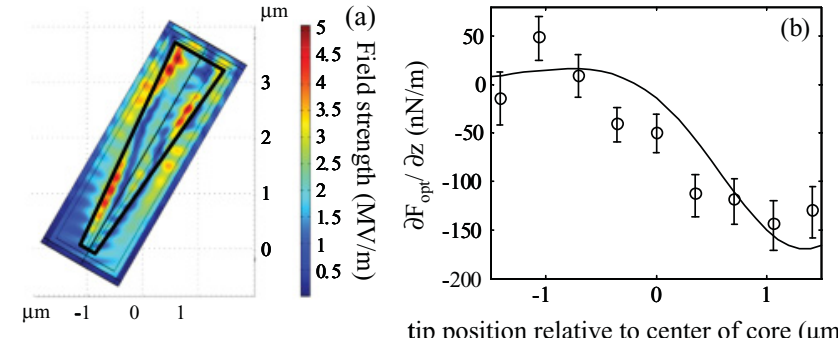

tip position relative to center of core $(\mu \mathrm{m})$

FIG. 3. (Color online) (a) Simulated probe geometry together with the electric-field distribution and (b) the calculated (solid line) and measured (symbols) gradients of the optical force acting on a probe scanning the core of the fiber along the direction indicated in the inset of Fig. 1(b). The error bars account for the errors associated with estimating $z$ and $A$ along with the noise in the topography maps.
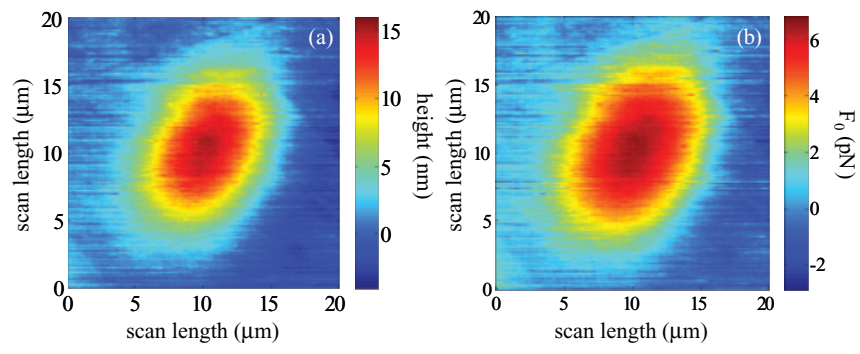

FIG. 4. (Color online) (a) Topography and (b) extracted optically induced force at the surface of a prism due to a focused laser beam illuminated in a TIR condition.

of the full electromagnetic calculation for different positions of the probe scanned in the direction indicated by the arrow in the inset of Fig. 1(b). We observe excellent quantitative agreement between the simulated and measured cases. The main sources of experimental errors are due to the accuracy in determining $z$ and $A$ as well as the noise in the topography signals.

In the experiment described above we demonstrated that optical forces can be measured with NSOM probes scanned through a complex optical field. However, the main contributions are due to homogeneous field components. To investigate the possibility of measuring optically induced forces in arbitrary fields, we conduct a second experiment where the optical field is evanescent and is thus measured in a more traditional scanning probe configuration. Here we use a standard pulled fiber NSOM probe with a $100-\mathrm{nm}$ aperture that is coated with a $10-\mathrm{nm} \mathrm{Cr}$ adhesion layer and a 250-nm-thick gold coating. This tip is scanned over the focused spot of a 532-nm laser illuminating a triangular prism in a total internal reflection (TIR) configuration.

To compute the optically induced force, the analysis described above may be repeated except that now Eq. (4) should be replaced with

$$
\begin{gathered}
F_{0} e^{-\alpha(z+\delta z)}\left(M_{1}^{0.5}(-2 \alpha A)-M_{2}^{1.5}(-2 \alpha A)\right) \\
=B_{L} R\left(\frac{1}{z^{n}} F_{W}^{n}-\frac{1}{(z+\delta z)^{n}} F_{W-\text { opt }}^{n}\right)
\end{gathered}
$$

to account for the exponential decay of the radiation from the surface [11]. $M_{a}^{b}(z)$ in Eq. (5) is the so-called Kummer function [11], $F_{0}$ is the force at the surface of the prism, $\alpha=2 k \sqrt{n^{2} \sin ^{2} \theta_{i}-1}$ is the decay constant, and $\theta_{i}$ is the angle of incidence at the top surface of the prism. From the experimental measurements we find $\alpha$ to be $0.00805 \mathrm{~nm}^{-1}$, which correlates very well with $\alpha=0.00836 \mathrm{~nm}^{-1}$ obtained from the above formula for $\theta_{i} \approx 45^{\circ}$ used in experiment. This confirms the electromagnetic origin of measured forces. The constants $B_{L}$ and $n$ in Eq. (5) are estimated based on the results in Ref. [19] where the force between an Au sphere in air and a silica plane is calculated using the Lifshitz theory. As can be seen from Eq. (5), in the case of evanescent fields the optical force itself is measured rather than its gradient. This occurs because the derivative of an exponential (evanescent decay) is also an exponential.

In Fig. 4(a) we show the measured topography when about $75 \mathrm{~mW}$ of laser light was focused onto the prism surface at the angle $\theta_{i} \approx 45^{\circ}$ resulting in a maximum intensity of approximately $5 \mathrm{~mW} / \mu \mathrm{m}^{2}$ at the surface. The spot size of the 
illumination (full width at half maximum) is about $13.2 \mu \mathrm{m}$ along the long axis. One can clearly see the influence of the optically induced force, even for the moderate intensity used here. Repeating the scan with the light turned off (not shown) reveals a flat surface with a rms roughness less than $1 \mathrm{~nm}$. The optically induced force at the prism surface, $F_{0}$, as evaluated from Eq. (5), is shown in Fig. 4(b).

We can compare again the experimental results with the estimations of our full electromagnetic calculations which account for a realistic tip model. A 1- $\mu$ m-long portion of the probe is considered. Because the optical field rapidly decreases away from the surface, this is sufficient to obtain a precise force estimation. The optically induced forces acting on the probe are calculated using Maxwell's stress tensor. We find that the force at the center of the illumination spot is $F_{0}^{\text {theor }} \approx 1.6 \mathrm{pN}$. This value is somewhat smaller the experimentally measured force of $F_{0}^{\exp } \approx 6 \pm 2 \mathrm{pN}$ extracted using the procedure outlined before. Note that, because practically only the very tip of the probe interacts with the optical radiation, this force simply scales with intensity across the illumination spot. One of the reasons for the difference between the values of the forces calculated numerically and measured experimentally may be our approximation of the NSOM probe as a solid Au sphere for the calculation of the van der Waals forces. Another possible aspect could be the influence of thermal (photophoretic) forces that can arise because of resistive heating in the metal coating on the probe. In order to assess the potential role of thermal effects we perform a transient heat transfer analysis using COMSOL. Calculations show that the stationary regime for the temperature distribution at the tip is achieved within $2 \mathrm{~ms}$ after exposure to the illumination. The temperature at the tip rises on average by $30 \mathrm{~K}$ and oscillates with amplitude of about $9 \mathrm{~K}$ following the mechanical oscillations of the probe. As our measurement technique is sensitive not to the absolute magnitude of the force but to the change of the force over the probe's oscillation cycle, these small temperature oscillations may have a minor contribution to the measured force $F_{0}^{\exp }$.

In conclusion, we presented a way to sense properties of both propagating and evanescent electromagnetic fields.
By measuring the mechanical action exerted on a standard scanning probe, one can acquire maps of the optically induced topography from which the gradient of the optical force can be evaluated. Notably, if such maps are collected at different intensities, the tip-sample separation can be determined without employing additional hardware. The approach permits quantitative measurements as demonstrated by the good agreement with full electrodynamics calculations.

In the case of the detection of propagating fields, we demonstrated a different modality to measure optically induced forces, which allows for the direct detection of the gradient of the force. In addition, we have shown that the influence of optical forces is significant for standard NSOM probes even when illuminated by evanescent waves at moderate intensities. Therefore, the effect of optical forces should be carefully considered in the practice of standard NSOM measurements.

Because the optical field is not actually coupled into the probe, the spatial resolution is not limited by the size of the aperture one can practically use. This is the major advantage of s-NSOM measurements, which we obtain without using additional optics, detection equipment, and the signal processing necessary to suppress the background scattering. In particular, the fact that a photon detector is not required opens up the possibility to measure radiation that is not readily detectable with standard detectors, e.g., infrared or tetrahertz radiation.

While the measurements presented in this paper were conducted with the feedback on such that the information about optical force was embedded in the topography signal, we could have alternatively maintained a constant tip-sample separation and used the phase signal in order to extract the same information. Such an extension is straightforward and may be used to determine the optical force while the probe remains close to the surface. Moreover, a similar measurement and data analysis can be performed for fields in free space without the presence of a material interface.

This work was partially supported by the Air Force Office of Scientific Research and by the Army Research Office.
[1] P. Lebedev, Ann. Phys. (Leipzig) 6, 433 (1901).

[2] E. F. Nichols and G. F. Hull, Phys. Rev. 13, 307 (1901).

[3] B. Cappella and G. Dietler, Surf. Sci. Rep. 34, 1 (1999).

[4] E. Meyer, H. J. Hug, and R. Bennewitz, Scanning Probe Microscopy: The Lab on a Tip (Springer, New York, 2004).

[5] C. Girard, A. Dereux, and O. J. F. Martin, Phys. Rev. B 49, 13872 (1994).

[6] X. Zhu, Y. Ling, G.-S. Huang, H.-T. Zhou, Y.-D. Dai, K. Wu, and Z.-Z. Gan, Chin. Phys. Lett. 15, 165 (1998).

[7] X. Zhu, G.-S. Huang, H.-T. Zhou, X. Yan, Z. Wang, Y. Ling, Y.-D. Dai, and Z.-Z. Gan, Opt. Rev. 4, 236 (1997).

[8] N. Satoh, T. Fukuma, K. Kobayashi, S. Watanabe, T. Fujii, K. Matsushige, and H. Yamada, Appl. Phys. Lett. 96, 233104 (2010).

[9] M. Abe, Y. Sagawara, K. Sawada, Y. Andoh, and S. Morita, Jpn, J. Appl. Phys. 37, L1074 (1998).

[10] A. Dogariu and R. Rajagopalan, Langmuir 16, 2770 (2000).
[11] F. J. Giessibl and H. Bielefeldt, Phys. Rev. B 61, 9968 (2000).

[12] G. L. Klimchitskaya, U. Mohideen, and V. M. Mostepanenko, Rev. Mod. Phys. 81, 1827 (2009).

[13] D. Tabor and R. H. S. Winterton, Proc. R. Soc. London Ser. A 312, 435 (1969).

[14] H.-J. Butt, B. Cappella, and M. Kappl, Surf. Sci. Rep. 59, 1 (2005).

[15] S. T. Huntington, P. G. Hartley, and J. Katsifolis, Ultramicroscopy 94, 283 (2003).

[16] S. C. Clark, J. Y. Walz, and W. A. Ducker, Langmuir 20, 7616 (2004).

[17] J. D. Jackson, Classical Electrodynamics, 3rd ed. (Wiley, New York, 1999).

[18] D. Haefner, S. Sukhov, and A. Dogariu, Phys. Rev. Lett. 103, 173602 (2009).

[19] P. J. van Zwol, G. Palasantzas, and J. Th. M. DeHosson, Phys. Rev. E 79, 041605 (2009). 